\title{
TRIM59 as a novel molecular biomarker to predict the prognosis of patients with NSCLC
}

\author{
MING LOU ${ }^{1,2}$, ZHAOJIA GAO $^{1}$, TAO ZHU $^{1}$, XIAOLIANG MAO ${ }^{1}$, \\ YEMING WANG $^{1}$, KAI YUAN ${ }^{1,2}$ and JICHUN TONG ${ }^{1}$
}

Departments of ${ }^{1}$ Thoracic Surgery, and ${ }^{2}$ Heart and Lung Disease, The Affiliated Changzhou No. 2 People's Hospital of Nanjing Medical University, Changzhou, Jiangsu 213003, P.R. China

Received March 31, 2019; Accepted November 1, 2019

DOI: $10.3892 / \mathrm{ol} .2019 .11199$

\begin{abstract}
As a member of the tripartite motif family, tripartite motif-containing protein 59 (TRIM59) serves as an E3 ubiquitin ligase in various cellular processes, including intracellular signaling, development, apoptosis, protein quality control, innate immunity, autophagy and carcinogenesis. The present study aimed to investigate the expression and prognostic value of TRIM59 in patients with non-small cell lung cancer (NSCLC). Expression of TRIM59 in patients with NSCLC was measured by immunohistochemistry in tissue microarrays. Datasets from The Cancer Genome Atlas (TCGA) were used to further verify the expression level of TRIM59 in NSCLC, lung adenocarcinoma and lung squamous cell carcinoma (LUSC). The prognostic value of TRIM59 in NSCLC was also analyzed. Immunohistochemistry revealed that TRIM59 was primarily located in the cytoplasm of tumor cells. Analysis of TCGA datasets revealed that TRIM59 was more highly expressed in tumor tissues than in normal tissues $(\mathrm{P}<0.0001)$. Furthermore, the TRIM59 expression level was associated with tumor differentiation $(\mathrm{P}=0.012)$, while no association was observed between TRIM59 expression and any other clinicopathological parameters. However, the average overall survival rate of patients with NSCLC in the high TRIM59 expression group was significantly lower than that in the low expression group $(\mathrm{P}=0.014)$, especially in patients with LUSC $(\mathrm{P}=0.016)$ and patients with poor differentiation $(\mathrm{P}=0.033)$. The multivariate analysis indicated that high TRIM59 expression is an independent prognostic factor in patients with NSCLC $(\mathrm{P}=0.018)$ and was associated with poor prognosis in patients with NSCLC. Therefore, TRIM59 may
\end{abstract}

Correspondence to: Dr Kai Yuan or Dr Jichun Tong, Department of Thoracic Surgery, The Affiliated Changzhou No. 2 People's Hospital of Nanjing Medical University, 29 Xinglong Lane, Changzhou, Jiangsu 213003, P.R. China

E-mail: yuankai1978@163.com

E-mail: tongjichun2012@163.com

Key words: non-small cell lung cancer, tripartite motif-containing protein 59, expression, prognosis serve as a novel molecular biomarker to predict the prognosis of patients with NSCLC.

\section{Introduction}

Lung cancer is associated with high mortality and its prevalence has increased worldwide in the past ten years $(1,2)$. Moreover, the incidence and mortality rates of lung cancer have increased significantly in China $(3,4)$. Histologically, non-small cell lung cancer (NSCLC) consists of several subtypes, including lung adenocarcinoma (LUAD), lung squamous cell carcinoma (LUSC), large cell lung cancer and other rarer types, of which LUAD and LUSC are the two main histological types (5). Although advances have been made in early diagnosis and systematic therapy, the 5-year survival rate for patients with NSCLC remains unsatisfactory (6). Therefore, investigating the pathogenesis of NSCLC may aid the identification of more sensitive and specific biomarkers as well as therapeutic targets for patients with NSCLC.

With the development of abundant open data resources, it is convenient and effective for researchers to screen cancer-related therapeutic targets (7). Through an analysis of The Cancer Genome Atlas (TCGA) database, the present study identified tripartite motif-containing protein 59 (TRIM59) as a novel lung cancer-related candidate target. The tripartite motif (TRIM) family of proteins comprises $>70$ members that are evolutionarily conserved and share RING, B-Box and coiled-coil (RBCC) multiple domains. TRIM proteins consist of a common $\mathrm{N}$-terminal Really Interesting New Gene (RING) finger domain, one or two B-box motifs and a coiled-coil region $(8,9)$. Due to the highly conserved RING domain, the majority of the proteins are E3 ubiquitin ligases that promote post-translational modifications of various substrates (10) and affect a range of cellular processes, including cell growth, development, differentiation, apoptosis, inflammation and immunity (11). TRIM59 not only participates in regulating protein expression, but also in the malignant behavior of tumor cells (12).

A number of studies have reported that TRIM59 acts as an oncogene in various types of cancer. Lin et al (13) reported that knockdown of TRIM59 inhibited tumor growth in prostate cancer. It was previously demonstrated that downregulation of TRIM59 inhibited proliferation, migration and invasion of breast cancer cells (14). Additionally, TRIM59 
induced epithelial-to-mesenchymal transition, and promoted migration and invasion of medulloblastoma cells through the phosphoinositide 3-kinase (PI3K)/protein kinase B (AKT) signaling pathway (15). However, to the best of our knowledge, there is little information available on the clinical significance and prognostic value of TRIM59 expression in NSCLC. The present study determined the expression of TRIM59 in NSCLC, and investigated its association with the occurrence, development and prognosis of NSCLC.

\section{Materials and methods}

TRIM59 expression in published TCGA databases. TCGA (https://www.cancer.gov/) is a collaboration between The National Cancer Institute (NCI) and The National Human Genome Research Institute, which rigorously control the quality of the datasets. Therefore, TCGA is a reliable source of information for disease analysis (16). To evaluate and analyze the TRIM59 expression level, three datasets, named LUNG exp_HiSeq-V2-2015-02-24, LUAD_exp_HiSeq-V2-2015-02-24 and LUSC_exp_HiSeq-V2-2015-02-24, were downloaded from the University of California, Santa Cruz Cancer Browser (https://genome-cancer.ucsc.edu). These datasets contain a list of cancer-related characteristic information of 1,013 NSCLC tissue samples, which include 108 paired NSCLC tissue samples, 57 pairs of LUAD tissues and 51 pairs of LUSC tissues. By analyzing the files named 'genomic Matrix' in these datasets, the mRNA expression levels of TRIM59 were obtained.

Patients and construction of a tissue microarray (TMA). In total, 140 NSCLC tissue and 10 normal adjacent tissue samples were obtained from patients who had undergone surgical resection at The Department of Thoracic Surgery of Zhongshan Hospital, Fudan University between January 2005 and December 2005. Complete clinical information was available for all patients (112 male and 28 female) and the mean age of the patients with NSCLC was 60.1 years (range, 26-79 years). Patients were classified according to the TNM classification system, formulated jointly by the American Joint Committee on Cancer and the Union for International Cancer Control (17). The clinical follow-up was recorded until July 2013. As previously described by Gao et al (18), the tissue samples from the 140 primary NSCLC cases and 10 normal adjacent lung tissues were arranged in rows and columns to construct a TMA. Patients involved in the present study had not received chemotherapy, radiotherapy or biotherapy before surgery.

Immunohistochemical staining and quantification analysis. The standard indirect immunoperoxidase procedures (Envision Plus; Dako; Agilent Technologies, Inc.) were adopted for immunohistochemistry to detect the expression of TRIM59 in NSCLC. Paraffin specimens were cut into slices (4- $\mu \mathrm{m}$ thick), which were mounted on slides, baked, deparaffinized and hydrated following conventional methods. Then, endogenous peroxidase activity was quenched by incubating the sections with $3 \% \mathrm{H}_{2} \mathrm{O}_{2}$ for $20 \mathrm{~min}$ at room temperature. Sections were then subjected to heat-induced antigen retrieval in $10 \mathrm{mM}$ citrate buffer ( $\mathrm{pH} 6.0$ ) for $10 \mathrm{~min}$ at $100^{\circ} \mathrm{C}$. Slides were incubated in $10 \mathrm{mM}$ TBS with $4 \%$ normal goat serum (Abcam) for $1 \mathrm{~h}$ at room temperature and incubated with the primary antibody against TRIM59(1:500; cat. no. YT4737; ImmunoWay Biotechnology Company) at $4{ }^{\circ} \mathrm{C}$ overnight. Following primary antibody incubation, the sections were washed with TBS and incubated with an HRP-conjugated goat anti rabbit secondary antibody (1:500; cat. no. RS0002; ImmunoWay Biotechnology Company) for $1 \mathrm{~h}$ at $37^{\circ} \mathrm{C}$. The sections were stained with DAB (3,3'-diaminobenzidine) (Dako; Agilent Technologies, Inc.) at $37^{\circ} \mathrm{C}$ for $10 \mathrm{~min}$ and weakly counterstained with hematoxylin at $37^{\circ} \mathrm{C}$ for $1 \mathrm{~min}$, dehydrated and covered with a coverslip. A light microscope (Nikon Corporation; magnification, x200) was used to acquire the images. Breast cancer tissue was used as a strong positive control for TRIM59 (19). For the negative control, PBS was added to the slides instead of primary antibody.

To quantify the expression of TRIM59 protein in NSCLC tissues, four fields in each section were selected for immunohistochemical scoring (magnification, x200). Two experienced pathologists assessed the immunohistochemical score independently. Based on a protocol developed by Xu et al (20), the intensity and proportion of positive tumor cells in the sections were used to calculate scores. The cytoplasmic staining intensity was scored as follows: i) 0 (no staining); ii) 1 (weak staining, light yellow); iii) 2 (moderate staining, yellow brown); and iv) 3 (strong staining, brown). The proportion of cytoplasmic positive cells was defined as $0-100 \%$ and was classified as follows: i) 1 (0-25\% positive cells); ii) $2(2-50 \%$ positive cells); iii) 3 (51-75\% positive cells); and iv) 4 (76-100\% positive cells). Finally, the staining intensity was multiplied by the proportion of positive cells to calculate the scores that were used to represent the expression levels of TRIM59. The higher the immunohistochemical scoring, the greater the expression of TRIM59. According to the scores, the 140 patients with NSCLC were classified into high expression (score $\geq 4$ ) and low expression (score <4) groups.

Statistical analysis. The $\chi^{2}$ test was used to investigate the association between TRIM59 expression and various clinicopathological parameters. The period from primary surgery until the death of the patient or the latest follow-up was defined as the overall survival (OS) time. Survival analysis was performed using the Kaplan-Meier method and differences were tested using a log-rank test. Univariate and Multivariate Wald test and cox proportional hazard regression models were constructed to identify the independent factors with a significant impact on patient OS time. A paired t-test was used to analyze TRIM59 mRNA expression in tumor and adjacent lung tissues. Statistical analyses were conducted using SPSS software (version 19.0; IBM, Corp.) and GraphPad Prism software (version 6.0; GraphPad Software, Inc.). $\mathrm{P}<0.05$ was considered to indicate a statistically significant difference. All results are expressed as the mean \pm standard deviation.

\section{Results}

Characteristics of the patients. The clinicopathological characteristics of the 140 patients with NSCLC in the present study are presented in Table I. The mean age of the patients was $60.1 \pm 6.4$ years (range, 26-79 years). The majority of the patients with NSCLC were male and $>50 \%$ of the tumors were LUSC. In total, there were 80 LUSC (grade 1-3), 46 LUAD (grade 1-3), 
Table I. Clinical and histological features of the 140 patients with NSCLC in the present study.

\begin{tabular}{|c|c|c|}
\hline Characteristic & $\begin{array}{l}\text { Number } \\
\text { of patients }\end{array}$ & $\%$ \\
\hline Age (years) ${ }^{\mathrm{a}}$ & $60(26-79)$ & - \\
\hline \multicolumn{3}{|l|}{ Sex } \\
\hline Male & 112 & 80 \\
\hline Female & 28 & 20 \\
\hline \multicolumn{3}{|l|}{ Location } \\
\hline Left & 64 & 46 \\
\hline Right & 76 & 54 \\
\hline \multicolumn{3}{|l|}{ Pathological type } \\
\hline Squamous cell carcinoma & 80 & 57 \\
\hline Adenocarcinoma & 46 & 33 \\
\hline Adenosquamous cell carcinoma & 5 & 4 \\
\hline Bronchiolo-alveolar carcinoma & 3 & 2 \\
\hline Sarcomatoid carcinoma & 1 & 1 \\
\hline Neuroendocrine carcinoma & 3 & 2 \\
\hline Mucoepidermoid carcinoma & 2 & 1 \\
\hline \multicolumn{3}{|l|}{ Differentiation } \\
\hline Poorly & 59 & 42 \\
\hline Moderate & 49 & 35 \\
\hline Well & 32 & 23 \\
\hline \multicolumn{3}{|l|}{ T stage } \\
\hline 1 & 15 & 11 \\
\hline 2 & 105 & 75 \\
\hline 3 & 20 & 14 \\
\hline \multicolumn{3}{|l|}{$\mathrm{N}$ stage } \\
\hline 0 & 82 & 59 \\
\hline 1 & 31 & 22 \\
\hline 2 & 27 & 19 \\
\hline \multicolumn{3}{|l|}{ NSCLC stage } \\
\hline I & 67 & 48 \\
\hline II & 44 & 31 \\
\hline III & 29 & 21 \\
\hline Follow-up period (months) ${ }^{\mathrm{a}}$ & $45(3-101)$ & - \\
\hline
\end{tabular}

${ }^{\text {aD }}$ ata are presented as the median (range). NSCLC, non-small cell lung cancer; $\mathrm{T}$, tumor; $\mathrm{N}$, node.

five adenosquamous cell carcinoma, three large-cell neuroendocrine carcinoma, three bronchiolo-alveolar carcinoma, two mucoepidermoid carcinoma, one sarcomatoid carcinoma and 10 normal lung tissues. The clinical follow-up was recorded until July 2013. There were 84 local recurrences or distant metastases and 91 deaths by the end of the follow-up period.

TRIM59 expression in NSCLC tissues. The immunohistochemical results showed that TRIM59 was mainly located in the cytoplasm of tumor cells, with low expression in normal lung tissue cells (Figs. 1 and 2). The average scores for all types of lung cancer tissues were 2-3-fold higher than normal lung tissue (Fig. 3A). The average scores for LUSC and LUAD were $3.93 \pm 2.82$ and $4.15 \pm 2.88$, respectively, while the normal lung score was $2.20 \pm 0.92$. Among the 140 NSCLC tissue samples, the high-TRIM59 expression group comprised 83 samples, which was significantly higher than that found in normal tissues (59.3 vs. $10.0 \%$; $\chi^{2}$ value, $9.201 ; \mathrm{P}=0.0024$; Fig. 3 ). To further verify this conclusion, TCGA datasets were analyzed. It was identified that TRIM59 was on average 1.32 -fold more highly expressed in NSCLC tissue samples compared with paratumour samples ( $\mathrm{P}<0.0001$; Fig. 3B). Similarly, TRIM59 was on average 1.24-fold more highly expressed in LUAD $(\mathrm{P}<0.0001$; Fig. 3C) and 1.40-fold more highly expressed in LUSC, compared with paratumor samples ( $\mathrm{P}<0.0001$; Fig. 3D).

TRIM59 expression and clinicopathological characteristics. The 140 patients with NSCLC were classified into low and high TRIM59 expression groups and the clinicopathological characteristics of the patients were compared. As shown in Table II, a significant association between TRIM59 expression and tumor differentiation was identified $(\mathrm{P}=0.012)$. However, no statistically significant associations were found between TRIM59 and tumor size $(\mathrm{P}=0.781)$, lymph node status $(\mathrm{P}=0.684)$, tumor stage $(\mathrm{P}=0.457)$ or any other clinicopathological characteristics.

Prognostic value of TRIM59 in NSCLC. A long-term follow-up was conducted to examine the association between TRIM59 expression and prognosis for patients with NSCLC. The average OS time of patients in the high and low TRIM59 expression groups were $46.586 \pm 4.011$ and $63.378 \pm 4.994$, respectively ( $\mathrm{P}=0.014$; Fig. $4 \mathrm{~A})$. Moreover, patients with poor differentiation had worse prognosis ( $\mathrm{P}=0.033$; Fig. 4B). Furthermore, Kaplan-Meier survival analyses of LUSC and LUAD were conducted. The results revealed that high expression of TRIM59 was significantly associated with worse OS time in patients with LUSC $(\mathrm{P}=0.016$; Fig. $5 \mathrm{~A})$, whereas no association was detected in patients with LUAD $(\mathrm{P}=0.196$; Fig. 5B). Finally, univariate and multivariate analyses were performed. As shown in Table III, high TRIM59 expression and lymph node metastasis had independent prognostic values $(\mathrm{P}=0.018$ and $\mathrm{P}=0.038$, respectively).

\section{Discussion}

The TRIM family consists of a series of highly conserved motif proteins with three domains that include a RING finger, one or two B-box motifs and a coiled-coil region (21). Due to the variability of the C-terminal domain of TRIM, the TRIM family proteins are further divided into 11 subfamilies $(22,23)$. In the past decade, the role of TRIM proteins in the innate immunity response to viral infection has attracted increasing attention (24-26). Previous studies have demonstrated that TRIM proteins are involved in several cell functions and participate in the process of ubiquitination as E3 ubiquitin ligases $(27,28)$. For example, TRIM59 was revealed to regulate autophagy by regulating the transcription and ubiquitination of beclin 1, which in turn affected the progression of NSCLC (29). Additionally, Zhou et al (30) reported that TRIM59 is upregulated in gastric cancer, and promotes the ubiquitination and degradation of p53 that affects tumor 
A
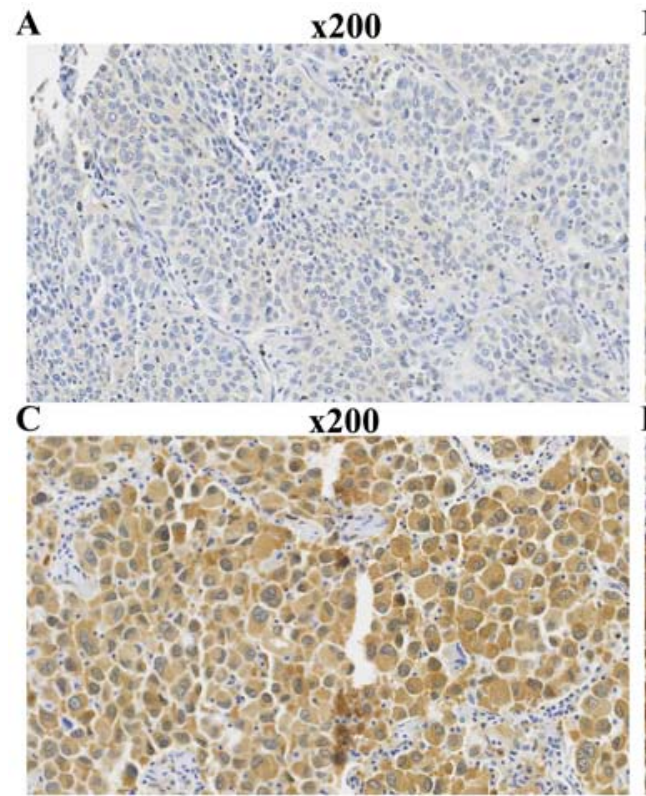

B $\quad \mathbf{2 0 0}$

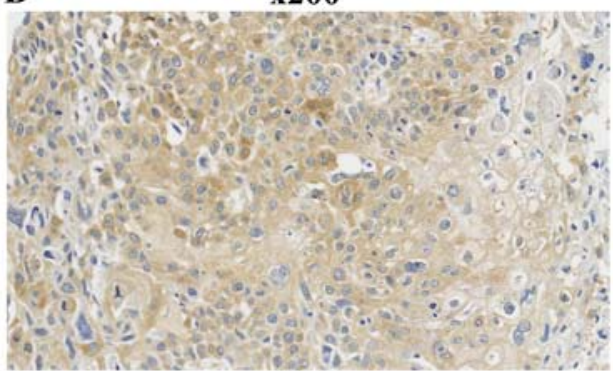

D

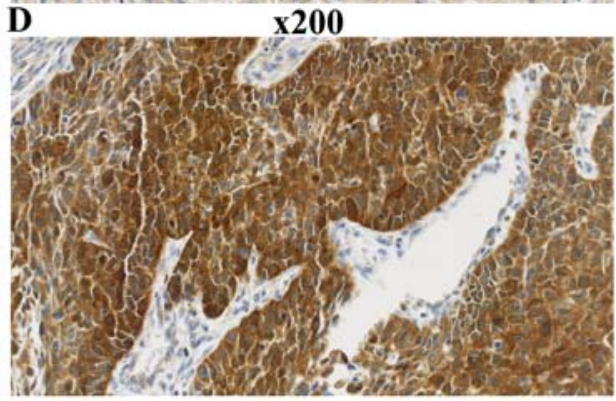

Figure 1. Representative immunohistochemical staining images of TRIM59 expression in 140 NSCLC tissues. TRIM59 was mainly expressed in the cytoplasm of tumor cells. (A) No staining, (B) weak staining, (C) moderate staining and (D) strong staining. Magnification, x200. TRIM59, tripartite motif-containing protein 59 .

A

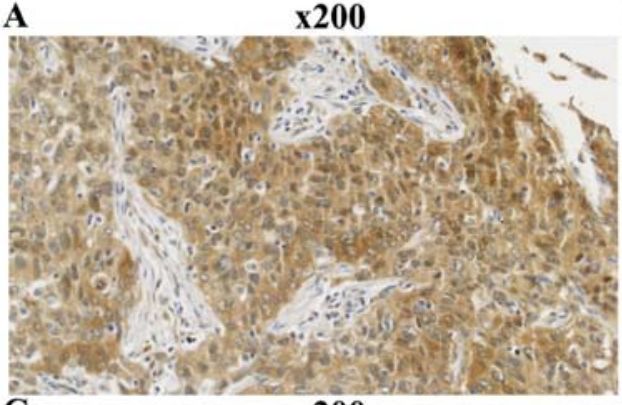

$\mathbf{C}$

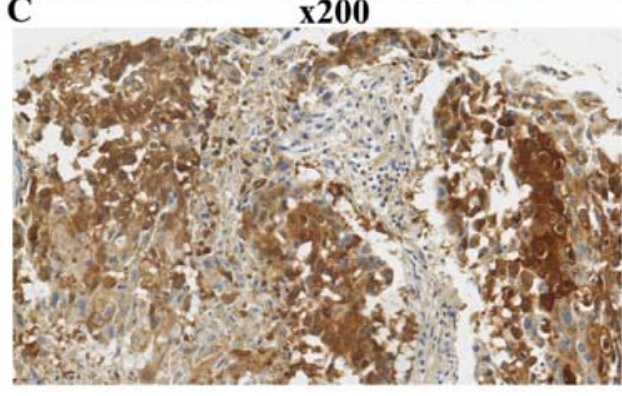

B
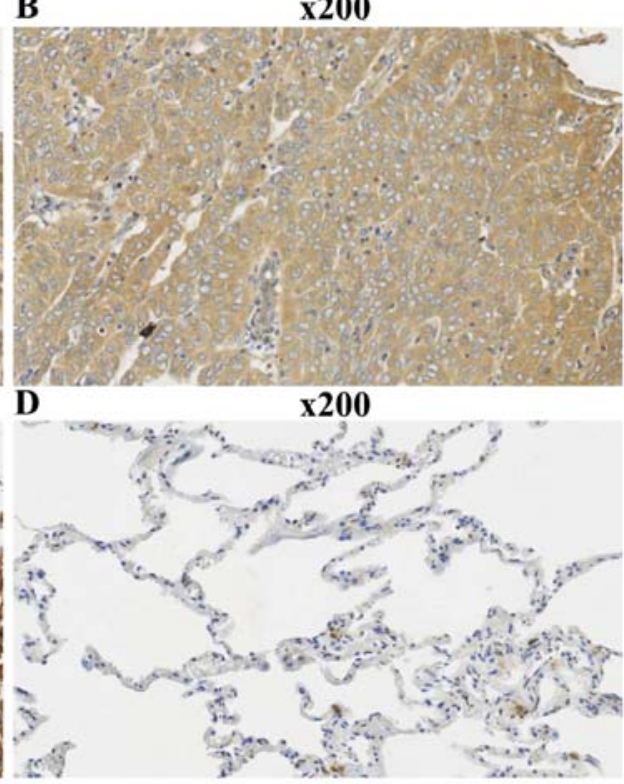

Figure 2. Immunohistochemical staining of TRIM59 in NSCLC and normal adjacent lung tissues. (A) LUSC, (B) LUAD, (C) breast cancer (as a strong positive control for TRIM59) and (D) normal adjacent lung tissues. Magnification, x200. TRIM59, tripartite motif-containing protein 59; NSCLC, non-small cell lung cancer; LUSC, lung squamous cell carcinoma; LUAD, lung adenocarcinoma.

growth. Ubiquitination, as a post-translational modification, is involved in many cellular processes, including signal transduction, protein quality control, transcription, cell cycle, apoptosis and development (31).

Certain TRIM proteins play important roles in the occurrence and development of NSCLC. For example, higher expression of TRIM29 was associated with worse prognosis in NSCLC (32). Dai et al (33) reported that knockdown of TRIM66 inhibited malignant behavior and epithelial-mesenchymal transition in NSCLC cells. Additionally, Luo et al (34) reported that TRIM44 promoted NSCLC development through activation of $\mathrm{NF}-\kappa \mathrm{B}$ signaling by upregulating $\mathrm{C}-\mathrm{X}-\mathrm{C}$ motif chemokine 16 and matrix metalloproteinase 9 expression. Furthermore, overexpression of TRIM44 enhanced the migratory and invasive abilities of the lung cancer cell lines A549 and H441 (34). Previous studies reported that TRIM59 was associated with the genesis, development and prognosis of tumors $(12,15,35)$. For example, TRIM59 was upregulated and promoted cell proliferation, migration and invasion in human osteosarcoma (36). Furthermore, it was found that TRIM59 facilitated the proliferation of colorectal cancer and promoted metastasis 

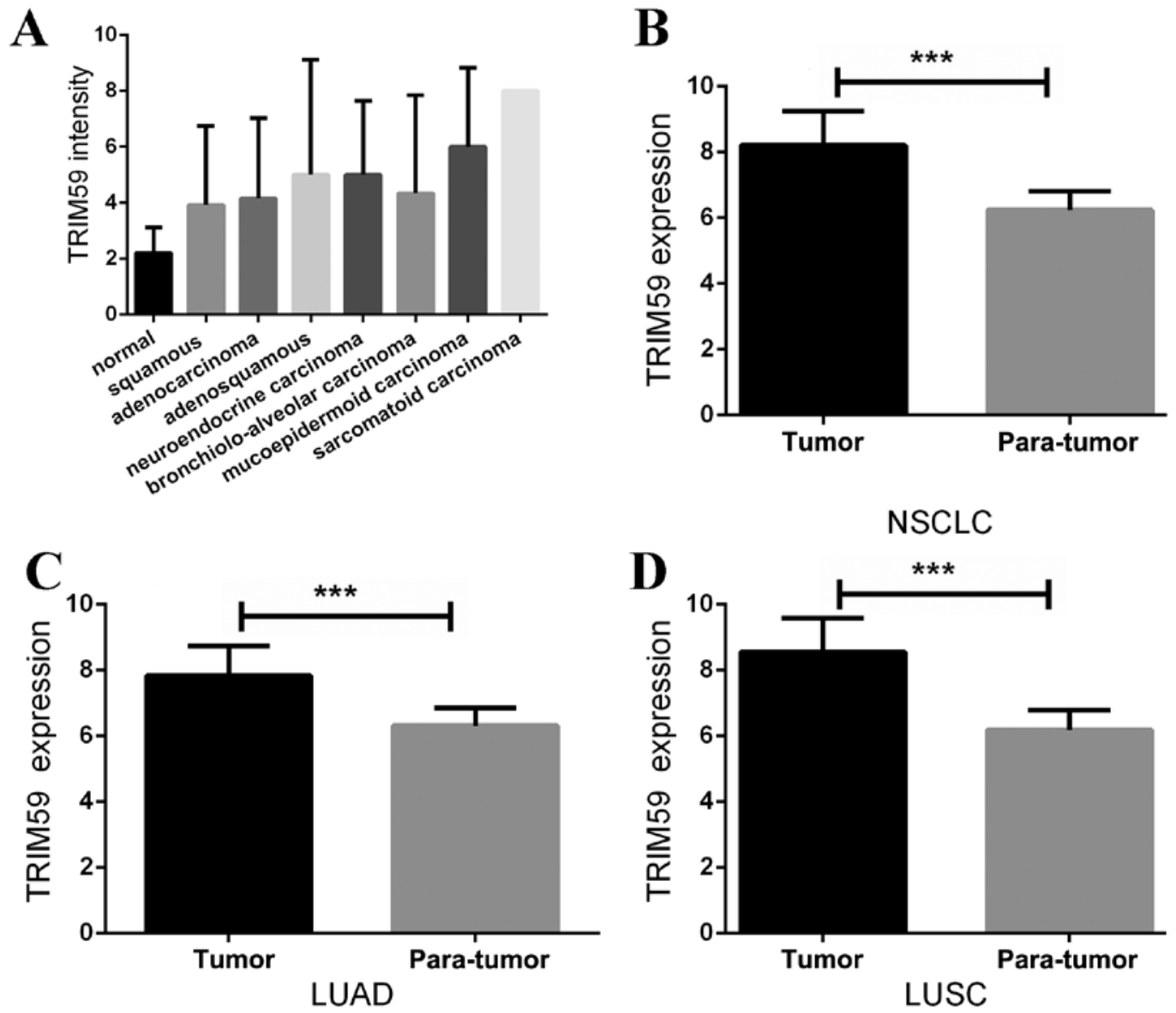

Figure 3. TRIM59 was more highly expressed in tumor tissues than in normal adjacent lung tissues. (A) Quantitative score of TRIM59 immunohistochemistry. In total, 108 pairs of NSCLC, 51 pairs of LUSC, 57 pairs of LUAD and 108 pairs of normal lung tissue specimens from TCGA datasets were examined to further verify the differential expression of TRIM59. TCGA datasets further verified that TRIM59 was 1.32-fold more expressed in NSCLC tissue samples; (B) 1.24-fold more expressed in LUAD; and (C) 1.40-fold more expressed in LUSC, compared with (D) in para-tumor samples. TRIM59, tripartite motif-containing protein 59; NSCLC, non-small cell lung cancer; LUSC, lung squamous cell carcinoma; LUAD, lung adenocarcinoma; TCGA, The Cancer Genome Atlas. ${ }^{* * *} \mathrm{P}<0.001$.

via the PI3K/AKT signaling pathway (37). A previous study revealed that upregulated TRIM59 served as a proto-oncogene and induced the progression of prostate cancer in transgenic mice (38). The upregulation of TRIM59 not only enhanced the expression of cyclin A, cyclin E, Bcl-xl, Bcl-2 and phosphorylated-AKT, and downregulated the expression of p21, p27 and p53, resulting in poor prognosis in breast cancer, but also affect breast cancer progression via the AKT signaling pathway (12). The aforementioned studies suggested that TRIM59 regulates a diverse range of cellular functions during tumor progression. However, its role in determining prognosis in NSCLC has not been fully established.

A previous study reported that TRIM59 expression was significantly increased in various NSCLC cell lines in vitro; however, data on the expression of TRIM59 in vivo was lacking. The present study investigated the expression profile and the prognostic value of TRIM59 in NSCLC. The expression of TRIM59 in patients with NSCLC was examined using an immunohistochemical method. Similar to a previous study by Khatamianfar et al (39), it was identified that TRIM59 may be a novel multiple cancer biomarker for the immunohistochemical detection of tumorigenesis. In the present study, TRIM59 was highly expressed in the majority of NSCLC tissues and was mainly expressed in the cytoplasm of tumor cells. TCGA datasets were used to further verify this result. Similar results were obtained in TCGA datasets, in which the expression of TRIM59 was significantly different between normal and tumor tissues. Based on this differential expression, it was hypothesized that there are functional roles associated with TRIM59. The patients in the present study were divided into high and low groups based on TRIM59 expression. It was observed that the TRIM59 expression level was associated with tumor differentiation, while no association was observed between TRIM59 expression and any other clinicopathological characteristics. Furthermore, it was identified that patients with high expression of TRIM59 had a worse prognosis than those with low TRIM59 expression, especially in patients with LUSC and patients with poor differentiation. Consistent with the results of a study performed by Zhan et al (40), TRIM59 promoted the proliferation and migration of NSCLC cells by upregulating cell cycle-related proteins, which may affect the prognosis of the patients with NSCLC. Additionally, the multivariate analysis performed in the present study indicated that high TRIM59 expression is an independent prognostic factor for patients with NSCLC. Collectively, the results suggested that TRIM59 functions as an oncogene in NSCLC and is associated with the genesis and development of NSCLC. 
Table II. Association between TRIM59 expression and clinicopathological factors.

\begin{tabular}{|c|c|c|c|}
\hline Clinicopathological factor & Low TRIM59 expression & High TRIM59 expression & P-value \\
\hline Age (years) & $59.8 \pm 10.4$ & $60.3 \pm 9.9$ & 0.265 \\
\hline \multicolumn{4}{|l|}{ Sex } \\
\hline Male & 46 & 66 & \multirow[t]{2}{*}{0.863} \\
\hline Female & 11 & 17 & \\
\hline \multicolumn{4}{|l|}{ Location } \\
\hline Left & 23 & 41 & \multirow[t]{2}{*}{0.291} \\
\hline Right & 34 & 42 & \\
\hline \multicolumn{4}{|l|}{ Histological type ${ }^{a}$} \\
\hline Squamous & 34 & 46 & \multirow[t]{2}{*}{0.896} \\
\hline Adenocarcinoma & 19 & 27 & \\
\hline \multicolumn{4}{|l|}{ Differentiation } \\
\hline Poorly & 26 & 33 & \multirow{3}{*}{0.012} \\
\hline Moderate & 25 & 24 & \\
\hline Well & 6 & 26 & \\
\hline \multicolumn{4}{|l|}{$\mathrm{pT}$} \\
\hline 1 & 7 & 8 & \multirow{3}{*}{0.781} \\
\hline 2 & 41 & 64 & \\
\hline 3 & 9 & 11 & \\
\hline \multicolumn{4}{|l|}{$\mathrm{pN}$} \\
\hline 0 & 35 & 47 & \multirow{3}{*}{0.684} \\
\hline 1 & 13 & 18 & \\
\hline 2 & 9 & 18 & \\
\hline \multicolumn{4}{|l|}{ TNM stage } \\
\hline I & 28 & 39 & \multirow{3}{*}{0.457} \\
\hline II & 20 & 24 & \\
\hline III & 9 & 20 & \\
\hline
\end{tabular}

${ }^{a}$ Certain types of tumors, including adenosquamous, bronchiolo-alveolar carcinoma, sarcomatoid carcinoma, neuroendocrine carcinoma and mucoepidermoid carcinoma, were not included. TRIM59, tripartite motif-containing protein 59; TNM, Tumor-Node-Metastasis.
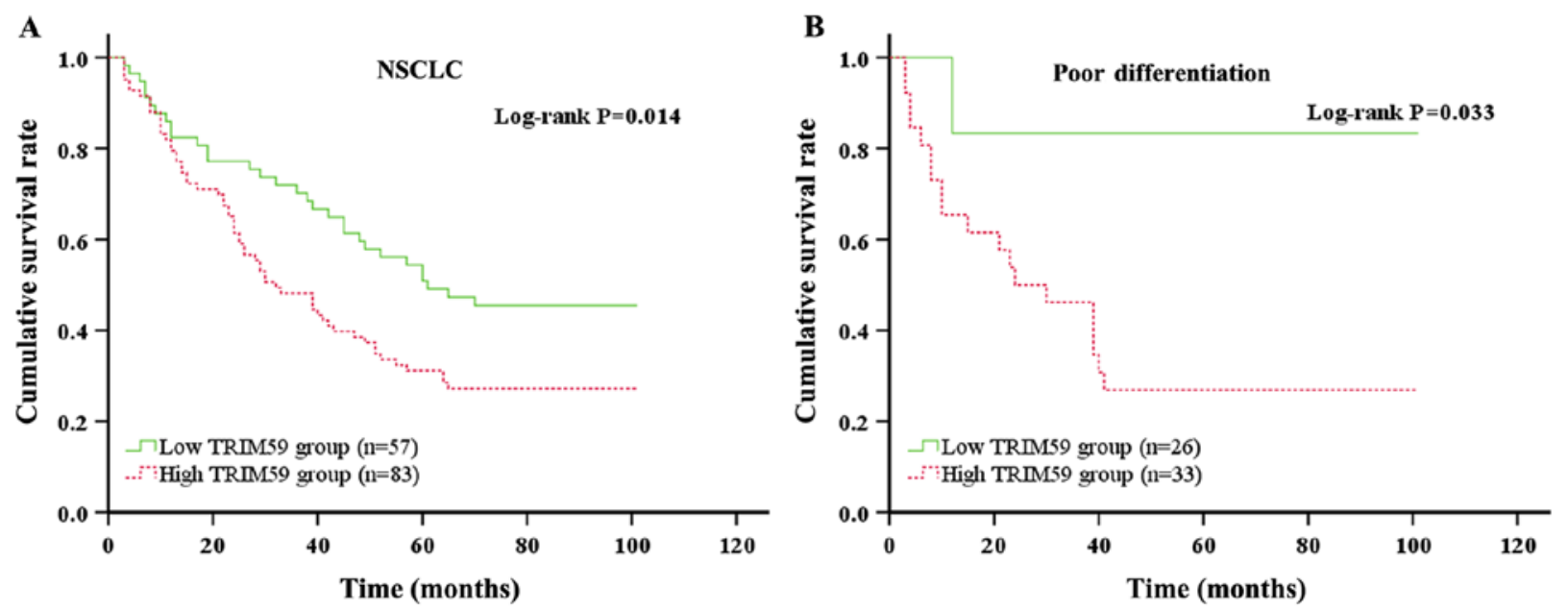

Figure 4. Kaplan-Meier survival estimates for (A) patients with NSCLC and (B) patients with poor differentiation. NSCLC, non-small cell lung cancer; TRIM59, tripartite motif-containing protein 59.

In conclusion, the present study revealed that high TRIM59 expression was associated with worse prognosis in patients with NSCLC and that TRIM59 may serve as an important prognostic biomarker in patients with NSCLC. 
Table III. Multivariate Cox regression analysis for potential factors influencing overall survival.

A, Univariate analysis

\begin{tabular}{|c|c|c|c|c|c|c|c|}
\hline \multirow[b]{2}{*}{ Parameter } & \multirow[b]{2}{*}{ B } & \multirow[b]{2}{*}{$\mathrm{SE}$} & \multirow[b]{2}{*}{ Wald } & \multirow[b]{2}{*}{ P-value } & \multirow[b]{2}{*}{ OR } & \multicolumn{2}{|c|}{$95 \% \mathrm{CI}$ for OR } \\
\hline & & & & & & Lower & Upper \\
\hline Age & 0.025 & 0.011 & 5.274 & 0.022 & 1.026 & 1.004 & 1.048 \\
\hline Sex & -0.240 & 0.275 & 0.762 & 0.383 & 0.786 & 0.458 & 1.349 \\
\hline Location & -0.216 & 0.210 & 1.059 & 0.303 & 0.806 & 0.534 & 1.216 \\
\hline Histological type & -0.123 & 0.213 & 0.337 & 0.562 & 0.884 & 0.583 & 1.341 \\
\hline Differentiation & -0.014 & 0.139 & 0.010 & 0.922 & 0.987 & 0.752 & 1.295 \\
\hline pT & 0.839 & 0.423 & 3.940 & 0.047 & 2.314 & 1.011 & 5.298 \\
\hline $\mathrm{pN}$ & 0.825 & 0.212 & 15.166 & 0.000 & 2.281 & 1.506 & 3.454 \\
\hline Stage & 0.655 & 0.215 & 9.275 & 0.002 & 1.926 & 1.263 & 2.937 \\
\hline TRIM59 & 0.535 & 0.222 & 5.799 & 0.016 & 1.708 & 1.105 & 2.640 \\
\hline
\end{tabular}

B, Multivariate analysis

\begin{tabular}{lcccccrcr}
\hline & & & & & & \multicolumn{2}{c}{$95 \%$ CI for OR } \\
\cline { 4 - 7 } Parameter & B & SE & Wald & P-value & OR & Lower & Upper \\
\hline Age & 0.021 & 0.011 & 3.928 & 0.047 & 1.022 & 1.000 & 1.043 \\
pT & 0.682 & 0.430 & 2.520 & 0.122 & 1.978 & 0.852 & 4.591 \\
pN & 0.802 & 0.386 & 4.304 & 0.038 & 2.229 & 1.045 & 4.754 \\
Stage & -0.040 & 0.395 & 0.010 & 0.919 & 0.961 & 0.443 & 2.083 \\
TRIM59 & 0.528 & 0.223 & 5.597 & 0.018 & 1.696 & 1.095 \\
\hline
\end{tabular}

B, regression coefficient; SE, standard error; Wald, Wald test; OR, odds ratio; p, pathological; T, tumor; N, node; CI, confidence interval; TRIM59, tripartite motif-containing protein 59.
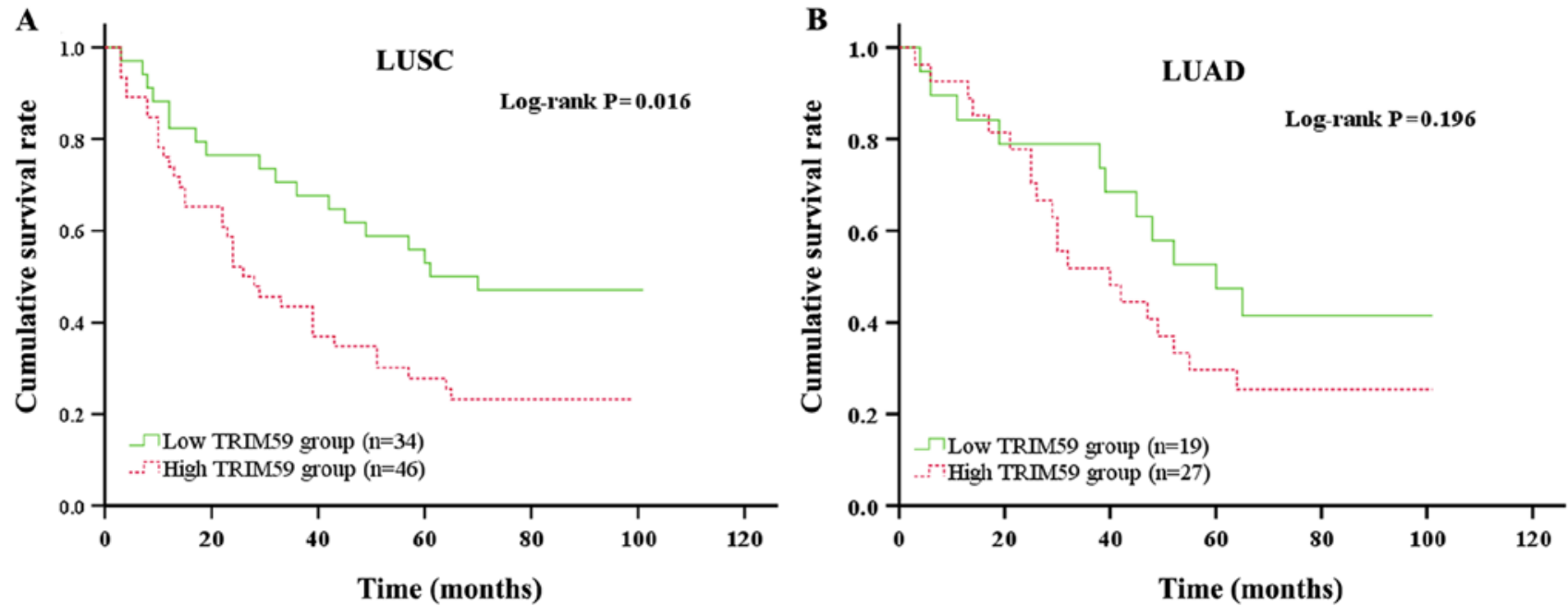

Figure 5. Kaplan-Meier survival estimates for (A) patients with LUSC and (B) patients with LUAD. LUSC, lung squamous cell carcinoma; LUAD, lung adenocarcinoma. TRIM59, tripartite motif-containing protein 59.

However, the present study had some limitations. A limited number of normal tissue samples were analyzed and the research was designed as a clinical retrospective study without investigating a specific mechanism. It was hypothesized that a TRIM59-related signaling pathway may downregulate TRIM59 expression and delay tumor progression. However, the exact mechanism of TRIM59 in NSCLC remains unclear and further research is required. 


\section{Acknowledgements}

Not applicable.

\section{Funding}

The present study was supported by The Medical Scientific Research Foundation of Jiangsu Commission of Health (grant no. H2018083), The High-Level Medical Talents Training Project (grant no. 2016CZBJ042) and The Jiangsu Provincial Medical Youth Talent [Jiangsu Health Scientific Education (2017; grant no. 3)].

\section{Availability of data and materials}

The datasets used or analyzed during the present study are available from the corresponding author on reasonable request. Moreover, datasets generated and/or analyzed during the current study are available in the TCGA repository (https://genome-cancer.ucsc.edu).

\section{Authors' contributions}

ML, KY and JT conceived and designed the experiments. ML and $\mathrm{ZG}$ performed the experiments. TZ and XM acquired data and contributed to reagents, materials and analysis tools. JT and YW analyzed the data. ML wrote the manuscript. KY and JT supervised the study. All authors read and approved the final manuscript.

\section{Ethics approval and consent to participate}

The present study was approved by The Research Ethics Committee of Zhongshan Hospital, Fudan University. All patients or their family members provided written informed consent.

\section{Patient consent for publication}

Not applicable.

\section{Competing interests}

The authors declare that they have no competing interests.

\section{References}

1. Siegel RL, Miller KD and Jemal A: Cancer statistics, 2017. CA Cancer J Clin 67: 7-30, 2017.

2. Hoffman RM and Sanchez R: Lung cancer screening. Med Clin North Am 101: 769-785, 2017.

3. Chen W, Zheng R, Zeng H and Zhang S: Epidemiology of lung cancer in China. Thorac Cancer 6: 209-215, 2015.

4. Sun KX, Zheng RS, Zeng HM, Zhang SW, Zou XN, Gu XY, Xia CF, Yang ZX, Li H, Chen WQ and He J: The incidence and mortality of lung cancer in China, 2014. Zhonghua Zhong Liu Za Zhi 40: 805-811, 2018 (In Chinese).

5. Mengoli MC, Longo FR, Fraggetta F, Cavazza A, Dubini A, Alì G, Guddo F, Gilioli E, Bogina G, Nannini N, et al: The 2015 world health organization classification of lung tumors: New entities since the 2004 classification. Pathologica 110: 39-67, 2018.

6. Hirsch FR, Suda K, Wiens J and Bunn PA Jr: New and emerging targeted treatments in advanced non-small-cell lung cancer. Lancet 388: 1012-1024, 2016.
7. Li CY, Xiong DD, Huang CQ, He RQ, Liang HW, Pan DH, Wang HL, Wang YW, Zhu HW and Chen G: Clinical value of miR-101-3p and biological analysis of its prospective targets in breast cancer: A study based on the cancer genome atlas (TCGA) and bioinformatics. Med Sci Monit 23: 1857-1871, 2017.

8. Ikeda $\mathrm{K}$ and Inoue S: TRIM proteins as RING finger E3 ubiquitin ligases. Adv Exp Med Biol 770: 27-37, 2012.

9. Hatakeyama S: TRIM family proteins: Roles in autophagy, immunity, and carcinogenesis. Trends Biochem Sci 42: 297-311, 2017.

10. Rajsbaum R, Garcia-Sastre A and Versteeg GA: TRIMmunity: The roles of the TRIM E3-ubiquitin ligase family in innate antiviral immunity. J Mol Biol 426: 1265-1284, 2014.

11. Chen W, Zhao K, Miao C, Xu A, Zhang J, Zhu J, Su S and Wang Z: Silencing Trim59 inhibits invasion/migration and epithelial-to-mesenchymal transition via TGF- $\beta / \mathrm{Smad} 2 / 3$ signaling pathway in bladder cancer cells. Onco Targets Ther 10: 1503-1512, 2017.

12. Liu Y, Dong Y, Zhao L, Su L, Diao K and Mi X: TRIM59 overexpression correlates with poor prognosis and contributes to breast cancer progression through AKT signaling pathway. Mol Carcinog 57: 1792-1802, 2018.

13. Lin WY, Wang H, Song X, Zhang SX, Zhou PS, Sun JM and Li JS: Knockdown of tripartite motif 59 (TRIM59) inhibits tumor growth in prostate cancer. Eur Rev Med Pharmacol Sci 20: 4864-4873, 2016.

14. Zhang Y and Yang WB: Down-regulation of tripartite motif protein 59 inhibits proliferation, migration and invasion in breast cancer cells. Biomed Pharmacother 89: 462-467, 2017.

15. Gao R, Lv G, Zhang C, Wang X and Chen L: TRIM59 induces epithelial-to-mesenchymal transition and promotes migration and invasion by PI3K/AKT signaling pathway in medulloblastoma. Oncol Lett 15: 8253-8260, 2018.

16. Yuan Y, Van Allen EM, Omberg L, Wagle N, Amin-Mansour A, Sokolov A, Byers LA, Xu Y, Hess KR, Diao L, et al: Assessing the clinical utility of cancer genomic and proteomic data across tumor types. Nat Biotechnol 32: 644-652, 2014.

17. Jin Y, Chen M and Yu X: Comparison of the 7(th) and proposed 8(th) editions of the AJCC/UICC TNM staging system for non-small cell lung cancer undergoing radical surgery. Sci Rep 6: 33587, 2016.

18. Gao ZJ, Wang Y, Yuan WD, Yuan JQ and Yuan K: HIF-2 $\alpha$ not HIF-1 $\alpha$ overexpression confers poor prognosis in non-small cell lung cancer. Tumour Biol 39: 1010428317709637, 2017.

19. Li XR, Ji F, Ouyang J, Wu W, Qian LY and Yang KY: Overexpression of RhoA is associated with poor prognosis in hepatocellular carcinoma. Eur J Surg Oncol 32: 1130-1134, 2006.

20. Xu H, Yu S, Yuan X, Xiong J, Kuang D, Pestell RG and Wu K: DACH1 suppresses breast cancer as a negative regulator of CD44. Sci Rep 7: 4361, 2017.

21. Esposito D, Koliopoulos MG and Rittinger K: Structural determinants of TRIM protein function. Biochem Soc Trans 45: 183-191, 2017.

22. Micale L, Chaignat E, Fusco C, Reymond A and Merla G: The tripartite motif: Structure and function. Adv Exp Med Biol 770: $11-25,2012$.

23. Streich FC Jr, Ronchi VP, Connick JP and Haas AL: Tripartite motif ligases catalyze polyubiquitin chain formation through a cooperative allosteric mechanism. J Biol Chem 288: 8209-8221, 2013.

24. Ozato K, Shin DM, Chang TH and Morse HC III: TRIM family proteins and their emerging roles in innate immunity. Nat Rev Immunol 8: 849-860, 2008.

25. McNab FW, Rajsbaum R, Stoye JP and O'Garra A: Tripartitemotif proteins and innate immune regulation. Curr Opin Immunol 23: 46-56, 2011

26. Chan E, Towers GJ and Qasim W: Gene therapy strategies to exploit TRIM derived restriction factors against HIV-1. Viruses 6: 243-263, 2014.

27. Tomar D and Singh R: TRIM family proteins: Emerging class of RING E3 ligases as regulator of NF- $\kappa$ B pathway. Biol Cell 107: 22-40, 2015.

28. Gushchina LV, Kwiatkowski TA, Bhattacharya $\mathrm{S}$ and Weisleder NL: Conserved structural and functional aspects of the tripartite motif gene family point towards therapeutic applications in multiple diseases. Pharmacol Ther 185: 12-25, 2018.

29. Han T, Guo M, Gan M, Yu B, Tian X and Wang JB: TRIM59 regulates autophagy through modulating both the transcription and the ubiquitination of BECN1. Autophagy 14: 2035-2048, 2018. 
30. Zhou Z, Ji Z, Wang Y, Li J, Cao H, Zhu HH and Gao WQ: TRIM59 is up-regulated in gastric tumors, promoting ubiquitination and degradation of p53. Gastroenterology 147: 1043-1054, 2014.

31. Watanabe $M$ and Hatakeyama S: TRIM proteins and diseases. J Biochem 161: 135-144, 2017.

32. Song X, Fu C, Yang X, Sun D, Zhang X and Zhang J: Tripartite motif-containing 29 as a novel biomarker in non-small cell lung cancer. Oncol Lett 10: 2283-2288, 2015.

33. Dai HY, Ma Y, Da Z and Hou XM: Knockdown of TRIM66 inhibits malignant behavior and epithelial-mesenchymal transition in non-small cell lung cancer. Pathol Res Pract 214: 1130-1135, 2018.

34. Luo Q, Lin H, Ye X, Huang J, Lu S and Xu L: Trim44 facilitates the migration and invasion of human lung cancer cells via the NF- $\mathrm{KB}$ signaling pathway. Int J Clin Oncol 20: 508-517, 2015.

35. Chen G, Chen W, Ye M, Tan W and Jia B: TRIM59 knockdown inhibits cell proliferation by down-regulating the Wnt/ $\beta$-catenin signaling pathway in neuroblastoma. Biosci Rep 39: pii: BSR20181277, 2019.

36. Liang J, Xing D, Li Z, Shen J, Zhao H and Li S: TRIM59 is upregulated and promotes cell proliferation and migration in human osteosarcoma. Mol Med Rep 13: 5200-5206, 2016.
37. Sun Y, Ji B, Feng Y, Zhang Y, Ji D, Zhu C, Wang S, Zhang C, Zhang D and Sun Y: TRIM59 facilitates the proliferation of colorectal cancer and promotes metastasis via the PI3K/AKT pathway. Oncol Rep 38: 43-52, 2017.

38. Valiyeva F, Jiang F, Elmaadawi A, Moussa M, Yee SP, Raptis L, Izawa JI, Yang BB, Greenberg NM, Wang F and Xuan JW: Characterization of the oncogenic activity of the novel TRIM59 gene in mouse cancer models. Mol Cancer Ther 10: 1229-1240, 2011.

39. Khatamianfar V, Valiyeva F, Rennie PS, Lu WY, Yang BB, Bauman GS, Moussa M and Xuan JW: TRIM59, a novel multiple cancer biomarker for immunohistochemical detection of tumorigenesis. BMJ Open 2: pii: e001410, 2012.

40. Zhan W, Han T, Zhang C, Xie C, Gan M, Deng K, Fu M and Wang JB: TRIM59 promotes the proliferation and migration of non-small cell lung cancer cells by upregulating cell cycle related proteins. PLoS One 10: e0142596, 2015.

This work is licensed under a Creative Commons Attribution-NonCommercial-NoDerivatives 4.0 International (CC BY-NC-ND 4.0) License. 